\title{
Indicadores Conductuales y Fisiológicos para Evaluar el Transporte de Novillos al Rastro y su Relación con el pH de la Carne
}

\author{
Behavioural and Physiological Indicators to Evaluate the Transport of Steers \\ TO THE Slaughterhouse AND ITS Relationship WITH PH OF THE BEEF \\ Marlyn Hellen Romero Peñuela ${ }^{1,2}$, Juan Velasco-Bolaños ${ }^{1}$, \\ Jorge Alberto Sánchez Valencia ${ }^{1}$
}

\section{RESUMEN}

El objetivo de este estudio fue evaluar el transporte de los novillos por medio de indicadores conductuales y fisiológicos, y su relación con el $\mathrm{pH}$ alto en la carne a las 24 horas (pH24) del sacrificio. Se evaluaron 56 novillos cebú ( $2.5 \pm 0.2$ años, $429.9 \pm 29.3 \mathrm{~kg}$ ) en cuatro viajes al centro de beneficio. El transporte se hizo bajo condiciones similares (ruta, camión y conductor), donde se evaluaron los indicadores conductuales (interacciones afiliativas y agonistas, posturas del cuerpo, dirección del cuerpo en el camión y exploración), indicadores fisiológicos de estrés y el $\mathrm{pH}_{24}$ de la carne. No se observaron diferencias significativas en las variables conductuales y el $\mathrm{pH}_{24}$ según los compartimentos del camión. Las interacciones afiliativas fueron más frecuentes que las agonistas. Las concentraciones sanguíneas del hematocrito, cortisol, glucosa y creatinaquinasa mostraron valores altos. El 63.5\% $(\mathrm{n}=36)$ de las carcasas presentaron $\mathrm{pH}_{24} \geq 5$.8. Los resultados indican que el transporte comercial con duración de $8 \mathrm{~h}$ fue un factor de estrés fisiológico para los novillos cebú, con implicaciones negativas sobre el alto $\mathrm{pH}$ cárnico. La ausencia de la mezcla social favoreció la cohesión y la estabilidad grupal.

Palabras clave: calidad de carne; bienestar animal; presacrificio; estrés

\section{AbStract}

The aim of the study was to evaluate the transport of steers by behavioral and physiological indicators and its relationship with high $\mathrm{pH}$ of the beef at $24 \mathrm{~h}$ after slaughter.

${ }^{1}$ Grupo de Investigación en Ciencias Veterinarias CIENVET, Programa de Medicina Veterinaria y Zootecnia, Departamento de Salud Animal, Facultad de Ciencias Agropecuarias, Universidad de Caldas, Manizales, Colombia

${ }^{2}$ E-mail: marlyn.romero@ucaldas.edu.co

Recibido: 21 de noviembre de 2016

Aceptado para publicación: 6 de abril de 2017 
A total of 56 Zebu steers $(2.5 \pm 0.2$ years old, $429.9 \pm 29.3 \mathrm{~kg})$ were evaluated in four trips to the slaughterhouse under similar conditions (route, truck, and driver), where behavioral indicators (affiliative and agonistic interactions, body postures, body direction in the truck and exploration), stress physiological indicators, and beef $\mathrm{pH}_{24}$ were evaluated. No significant differences were observed in the behavioral variables and the muscle $\mathrm{pH}_{24}$ according to the compartments of the truck. The affiliative interactions were more frequent than the agonistic. Hematocrit and cortisol, glucose and creatine kinase blood concentrations showed high values. The $63.5 \%(\mathrm{n}=36)$ of the carcasses presented $\mathrm{pH}_{24}$ $\geq 5$. . These results indicate that the commercial transport with duration of $8 \mathrm{~h}$ was a physiological stress factor for Zebu steers, with negative implications on high $\mathrm{pH}$ of the beef. The absence of the social mixture favored the cohesion and group stability.

Key words: meat quality; animal welfare; pre-slaughter; stress

\section{INTRODUCCIÓN}

El transporte representa una fase crítica en la producción ganadera y con frecuencia es considerado como una de las principales causas de estrés para los bovinos, que puede tener repercusiones negativas en el bienestar y en la salud de los animales (Cafazzo et al., 2012; Magnani et al., 2014). Los bovinos pueden expresar las consecuencias de los eventos estresantes mediante cambios fisiológicos y conductuales específicos. Las alteraciones conductuales son, a menudo, los primeros signos de estrés en los bovinos y esto, a su vez, se relaciona con las respuestas fisiológicas y con los atributos de calidad de la carne (Miranda-de la Lama, 2010; Stockman et al., 2013).

En la actualidad, el transporte de larga duración de bovinos en pie ha aumentado para responder a las exigencias del comercio internacional y por los beneficios económicos alcanzados (Lambooij et al., 2012). La duración del viaje es una de las variables de mayor interés para evaluar la calidad del transporte en bovinos de engorde. El tiempo de transporte prolongado puede ser un severo factor estresante que causa entre el $1.5 \mathrm{y}$ $9 \%$ de pérdida de peso vivo por la deshidratación y ausencia de alimento (Gallo et al., 2003); asimismo, aumenta el riesgo de caída, muerte y contusiones (Romero et al., 2013), aumento de la susceptibilidad a las infecciones (Cockram et al., 2004), pérdidas económicas por eliminación de tejido contuso, menor rendimiento en canal y descenso en la categoría de tipificación de las canales (Gallo et al., 2003), mayor riesgo de contaminación de la carne con microorganismos entéricos (Mather et al., 2007) y disminución en la calidad de la carne, por la presencia de la carne denominada corte oscuro o DFD (oscura, firme y seca, por sus siglas en inglés) (Romero et al., 2017).

En Colombia, el sector ganadero se encuentra en un proceso de modernización selectiva de las condiciones de producción y de la logística del presacrificio. Sin embargo, la ganadería bovina presenta una alta dispersión geográfica, porque cerca del $70 \%$ del ganado de carne es sacrificado en plantas ubicadas en centros de consumo, con la participación de un alto número de intermediarios (comisionistas y acopiadores, colocadores y subastas). Esto implica el traslado de los animales desde los centros productivos a través de vías de montaña, con un tiempo de transporte prolongado, y por medio de una infraestructura poco especializada y condiciones geográficas diversas (Romero et al., 2012). El objeto del presente trabajo fue evaluar el efecto de la localización en el camión (compartimento anterior y posterior) y otros 
aspectos del transporte comercial, con una duración de ocho horas, sobre la respuesta conductual y fisiológica de los bovinos, incluyendo el $\mathrm{pH}$ de la carne.

\section{Materiales y Métodos}

\section{Descripción del Estudio}

El estudio se realizó en noviembre y diciembre de 2012. Se evaluaron 56 bovinos machos castrados (novillos) manejados bajo condiciones comerciales, con características similares en cuanto a origen, raza (cruces comerciales de ganado cebú), edad ( $2.5 \pm$ 0.2 años) y peso vivo $(429.9 \pm 29.3 \mathrm{~kg})$. La granja estaba localizada en el valle del río Magdalena (150 msnm). Los novillos fueron cebados bajo condiciones extensivas en pasturas naturales, suministro de sal mineralizada y agua a voluntad. Los animales se identificaron individualmente con números sobre la piel utilizando un marcador y se mantuvieron en los mismos lotes de origen hasta el sacrificio.

El embarque se efectuó sin el uso de elementos contundentes. Se realizaron cuatro viajes bajo similares condiciones en cuanto a ruta, camión y conductor. La ruta utilizada comprendía un trayecto plano mixto $(150 \mathrm{~km}$, altitud promedio $158.8 \mathrm{msnm}$ ) y otro pavimentado de montaña $(125 \mathrm{~km}$, altitud máxima $3681 \mathrm{msnm}$ ). El vehículo fue el característico para el transporte de ganado en Colombia (con aforo de 14 novillos), de dos ejes con chasis rígido (combinado con madera y acero), provisto de ventilación pasiva, techo con carpa de lona y con una capacidad para 10 t. El camión se dividió en dos compartimentos en donde se ubicaron grupos de siete novillos, con una densidad de carga de $344 \mathrm{~kg} / \mathrm{m}^{2}$.

La planta de sacrificio contaba con una rampa de desembarque de concreto y pisos antideslizantes, que conectaban con una serie de corredores hasta los corrales de re- cepción. Estos tenían un área de $57.8 \mathrm{~m}^{2}$ (5.9 $\mathrm{m}$ de ancho y $9.8 \mathrm{~m}$ de largo), con pisos antideslizantes de concreto y techados. El desembarque se efectuó con el uso de interacciones visuales. Los novillos permanecieron en la planta durante $24 \mathrm{~h}$ en corrales de espera techados, con suministro de agua y sin mezcla social. Fueron aturdidos con pistolas de proyectil retenido, tipo no penetrante. Se recolectaron tres muestras en el desangrado, una con anticoagulante (EDTA), otra sin anticoagulante y la tercera con fluoruro de sodio (NaF) (glucosa y lactato).

La investigación fue aprobada por el Comité de Ética para la Experimentación con Animales (CEEA) de la Universidad de Caldas (Manizales, Colombia), mediante el Acta 1 del 13 de febrero de 2012 (actividades con riesgo mínimo).

\section{Medidas de Comportamiento}

Se elaboró un etograma seguido de una prueba piloto para registrar las conductas individuales y sociales de los novillos. Su comportamiento durante el viaje se grabó mediante un sistema de vídeo cámara a partir del momento en que el camión salió de la finca hasta la llegada a la rampa de desembarque de la planta de sacrificio. Las cámaras digitales se localizaron en cada compartimento del camión en un mismo ángulo y altura $(1.8 \mathrm{~m})$ y conectadas a un sistema de grabación DVR.

Los cuatro viajes fueron evaluados por un mismo observador entrenado, de acuerdo con los parámetros descritos en el Cuadro 1. Se utilizó la técnica de muestreo conductual de barrido con «registro instantáneo» cada 10 min para obtener información sobre las interacciones sociales (comportamientos afiliativos y agonistas) (Miranda-de la Lama et al., 2012). La orientación de los novillos durante el viaje (diagonal, perpendicular o paralelo al eje central del camión), las posturas corporales (pie, echado, caída y pérdida de balance) y el comportamiento exploratorio se registraron usando el método de muestreo 
Cuadro 1. Categorías conductuales evaluadas durante el transporte de novillos

\begin{tabular}{ll}
\hline Parámetros conductuales & Descripción \\
\hline $\begin{array}{c}\text { Comportamiento afiliativo } \\
\text { Lamer }\end{array}$ & El novillo lame a otro individuo \\
Olfatear & El novillo huele a otro individuo \\
Frotar & El animal talla su cabeza contra otro individuo \\
Apoyar & El novillo apoya su cabeza sobre el lomo de otro \\
individuo & \\
Comportamientos agonistas & El novillo hostiga a otro individuo sin ejercer contacto \\
Amenaza & El novillo contacta con su cabeza en el cuerpo de otro \\
individuo de forma agresiva & De pie, echado, caída, pérdida de balance por vibración \\
del vehículo & Perpendicular, diagonal o paralelo al eje horizontal del \\
camión & \\
Orienturas corporales & Olfateo del entorno, camión u objetos \\
\hline
\end{tabular}

Adaptado de Cafazzo et al. (2012)

instantáneo. Para el análisis, se agruparon las observaciones conductuales cada dos horas, hasta completar ocho horas de viaje.

\section{Variables Sanguíneas}

El análisis de las variables sanguíneas constó de la determinación del volumen globular acumulado (\%VGA) mediante la técnica de microhematocrito, el perfil de leucocitos mediante la observación microscópica de extendidos sanguíneos teñidos con el colorante Wright y cortisol plasmático $(\mu \mathrm{g} / \mathrm{dl})$ mediante radioinmunoanálisis (RIA) (Coeficiente de variación inter-ensayo: 9.3\%).

Asimismo, se determinaron las concentraciones de glucosa $(\mathrm{mmol} / \mathrm{l})$, urea $(\mathrm{mmol} / \mathrm{l})$, proteína total $(\mathrm{g} / \mathrm{l})$, creatinina $(\mathrm{mmol} / \mathrm{l})$, albúmina (g/l), y la actividad plasmática de la enzima creatinquinasa $(\mathrm{CK})(\mathrm{U} / \mathrm{l})$ usando el kit comercial de Biosystem $^{\circledR}$. Las concentraciones sanguíneas del $\beta$-hidroxibutirato $(\beta \mathrm{HB})$ $(\mathrm{mmol} / \mathrm{l})$ y el lactato $(\mathrm{mmol} / \mathrm{l})$, se establecieron usando el kit comercial de Randox ${ }^{\circledR}$. Todas las lecturas se efectuaron en el espectrofotómetro BTS-330 $\left(\right.$ Biosystem $\left.^{\circledR}\right)$.

\section{Determinación del pH}

El pH a las 24 horas del sacrificio $\left(\mathrm{pH}_{24}\right)$ se midió en el músculo longissimus thoracis expuesto a nivel de la quinta y sexta costilla del lado derecho de la canal a $4 \mathrm{~cm}$ de profundidad, con un potenciómetro de punzón para carnes (IQ150 pH/Mv/medidor de temperatura; IQ Scientific Intruments $\left.{ }^{\circledR}\right)$. El equipo fue calibrado cada cinco muestras, usando dos soluciones estándar de $\mathrm{pH} 4$ y 7 ; asimismo, se lavó con agua destilada después de cada medición. 
Cuadro 2. Cambios en las variables conductuales en novillos durante ocho horas de transporte en camión (Media $\pm \mathrm{EE}$ )

\begin{tabular}{lcccc}
\hline \multirow{2}{*}{ Variables conductuales } & \multicolumn{4}{c}{ Tiempo de transporte $(\mathrm{h})$} \\
\cline { 2 - 5 } & 2 & 4 & 6 & 8 \\
\hline Afiliativas & & & & \\
Frotar & $15.0 \pm 5.1$ & $10.1 \pm 5.1$ & $8.4 \pm 3.4$ & $9.5 \pm 4.1$ \\
Apoyar & $36.8 \pm 3.5^{\mathrm{a}}$ & $35.0 \pm 4.4^{\mathrm{a}}$ & $29.0 \pm 5.0^{\mathrm{a}}$ & $19.0 \pm 3.3^{\mathrm{b}}$ \\
Agonistas & & & \\
Amenaza & $0.6 \pm 0.1^{\mathrm{a}}$ & $1.3 \pm 0.5^{\mathrm{a}}$ & $2.1 \pm 0.8^{\mathrm{b}}$ & $2.3 \pm 0.5^{\mathrm{b}}$ \\
Topeteo & $1.3 \pm 0.5^{\mathrm{a}}$ & $2.4 \pm 0.8^{\mathrm{a}}$ & $4.6 \pm 0.8^{\mathrm{b}}$ & $4.0 \pm 0.5^{\mathrm{b}}$ \\
Posturas corporales & & & & \\
Echado & $0.2 \pm 0.1$ & $0.3 \pm 0.1$ & $0.5 \pm 0.3$ & $1.1 \pm 0.3$ \\
Pie & $6.9 \pm 0.3$ & $6.7 \pm 0.4$ & $6.6 \pm 0.5$ & $6.0 \pm 0.8$ \\
Caídas & $1.6 \pm 1.4^{\mathrm{a}}$ & $4.1 \pm 2.1^{\mathrm{b}}$ & $5.1 \pm 3.3^{\mathrm{b}}$ & $7.3 \pm 6.7^{\mathrm{c}}$ \\
Balance & $22.5 \pm 14.2^{\mathrm{a}}$ & $28.1 \pm 10.2^{\mathrm{a}}$ & $32.2 \pm 11.5^{\mathrm{b}}$ & $20.0 \pm 8.6^{\mathrm{a}}$ \\
Exploración & $30.0 \pm 11.8^{\mathrm{a}}$ & $22.3 \pm 7.7^{\mathrm{a}}$ & $18.3 \pm 5.3^{\mathrm{b}}$ & $11.0 \pm 5.0^{\mathrm{c}}$ \\
Orientación en el & & & & \\
camión & & & & \\
Paralelo & $0.3 \pm 0.1$ & $0.3 \pm 0.1$ & $0.5 \pm 0.2$ & $0.8 \pm 0.3$ \\
$\quad$ Perpendicular & $5.2 \pm 0.6$ & $5.1 \pm 0.4$ & $4.9 \pm 0.6$ & $4.2 \pm 0.6$ \\
Diagonal & $1.6 \pm 0.5$ & $1.7 \pm 0.4$ & $1.7 \pm 0.4$ & $2.1 \pm 0.5$ \\
\hline
\end{tabular}

$a, b, c$ Superíndices diferentes dentro de filas indican diferencia significativa $(p<0.05)$

\section{Análisis Estadístico}

El análisis se efectúo utilizando el programa Stata v. 12.0 (College Station, Texas, EEUU). Se estableció la distribución normal de las variables fisiológicas, conductuales y el $\mathrm{pH}$ por medio del análisis de los residuales. Las variables sanguíneas VGA, creatinina, BHB y el recuento total de leucocitos presentaron una distribución normal. Las concentraciones de CK, albúmina, lactato, urea, cortisol, glucosa y lactato se transformaron en su logaritmo natural. Este mismo procedimiento se efectuó para las variables conductuales. Los valores obtenidos fueron utilizados en los análisis posteriores. Todas las estimaciones en escala logarítmica fueron transformadas nuevamente y se presentaron en su escala original como medias \pm $\mathrm{DE}$ o medias $\pm \mathrm{EE}$. Se empleó la prueba TStudent para investigar si existían diferencias significativas entre las frecuencias de los variables conductuales y sanguíneas según la localización de los animales en el camión (compartimento anterior y posterior).

Los comportamientos afiliativos y agonistas totales, las posturas corporales y la orientación en el camión no presentaron una distribución normal y se estudiaron por me- 
dio de la distribución de Poisson de acuerdo con el tiempo de viaje transcurrido en intervalos de $2 \mathrm{~h}$ y la localización en el camión, y se ajustó el análisis para establecer si había diferencias entre los cuatro viajes evaluados. Se obtuvo el riesgo relativo (RR) para cada variable estudiada. Se evalúo la bondad de ajuste de los modelos obtenidos mediante la comparación de los gráficos de residuales frente a los valores del predictor lineal. Un valor de $\mathrm{p}<0.05$ fue aceptado como significativo en todos los análisis.

\section{Resultados y Discusión}

Los cambios en la frecuencia del comportamiento durante el transporte se utilizan para evaluar el estrés durante el viaje, toda vez que es una medida de la habilidad del ganado de enfrentar los desafíos de esta etapa y que, con frecuencia, representan el primer nivel de respuesta a condiciones adversas (Temple et al., 2011; Magnani et al., 2014). No se observaron diferencias significativas en las variables conductuales entre los novillos localizados en el compartimento anterior y posterior del camión, ni entre los viajes estudiados. Las interacciones afiliativas fueron más frecuentes que las agonistas, las cuales aumentaron levemente al final del viaje. Las pérdidas de balance y la exploración del ambiente por parte de los bovinos fueron prevalentes, pero esta última presentó una relación inversamente proporcional al tiempo de viaje (Cuadro 2).

Los comportamientos afiliativos son un indicador de las experiencias positivas de los bovinos en los sistemas productivos cuando se miden bajo condiciones comerciales (Boissy et al., 2007). La mayor frecuencia de interacciones afiliativas en los bovinos durante los viajes pudo ser consecuencia de la ausencia de mezcla social, que favoreció el mantenimiento de la cohesión y estabilidad del grupo (Millman y Duncan, 2001; Mounier et al., 2005). Estos comportamientos ayudan a reducir las agresiones y están asociados con emociones positivas que mejoran el nivel de bienestar animal (Miranda-de la Lama y Martiello, 2010; Miranda de la Lama et al., 2012).

La alta frecuencia de comportamientos exploratorios observados en este estudio, son considerados como expresiones de bajos niveles de miedo en el ganado (Lambooij et al., 2012). El bajo nivel de comportamiento agonista puede indicar que la novedad y la duración del transporte no fueron suficientes para que los bovinos reestablecieran relaciones de dominancia y jerarquía social en el grupo, aspecto también descrito en porcinos (Magnani et al., 2014); sin embargo, estos aumentaron levemente durante el viaje, porque en los grupos sociales los individuos con frecuencia experimentan algún tipo de amenaza de sus congéneres (Miranda-de la Lama y Martiello, 2010; Fox et al., 2014).

Los encuentros antagónicos pueden causar estrés y un incremento de las concentraciones de cortisol sérico (Mounier et $a l ., 2005)$, aspecto presente en este estudio. Se ha sugerido que las manifestaciones conductuales de los animales ante un agente estresante están de manera estrecha asociadas con el incremento de cortisol, debido a que sus receptores se encuentran localizados en regiones específicas involucradas con la regulación hormonal (hipotálamo e hipófisis) y, en particular, con el sistema límbico, que juega un papel relevante en las conductas emocionales. Es así que las concentraciones plasmáticas de cortisol y glucosa han sido usadas como un indicador confiable de estrés físico agudo y estrés emocional (Kannan et al., 2003).

La orientación perpendicular al eje del camión fue la más frecuente, porque facilita mantener el equilibrio. Así mismo, el ganado permaneció poco tiempo echado mientras que el vehículo estaba en movimiento, aspecto descrito por otros investigadores, tanto en trayectos de corta y larga duración (Cafazzo et al., 2012; Tarrant y Grandin, 2000). 
Cuadro 3. Resumen de los modelos de regresión de Poisson para variables de comportamiento de novillos durante viajes de ocho horas en camión

\begin{tabular}{lcccc}
\hline $\begin{array}{l}\text { Comportamiento/horas de } \\
\text { viaje }\end{array}$ & Coeficiente & $\begin{array}{c}\text { Error } \\
\text { estándar }\end{array}$ & RR $^{1}$ & Valor $p$ \\
\hline $\begin{array}{l}\text { Caídas } \\
\text { Horas de viaje }\end{array}$ & Ref. $^{2}$ & Ref. & Ref. & \\
$0-2$ & 0.93 & 0.32 & 2.5 & $<0.01$ \\
$2.1-4$ & 1.1 & 0.31 & 3.2 & $<0.01$ \\
$4.1-6$ & 1.5 & 0.30 & 4.5 & $<0.01$ \\
$6.1-8$ & & & & \\
Afiliativos (total) & & & & \\
Horas de viaje & Ref. & Ref. & Ref. & \\
$0-2$ & -0.13 & 0.07 & 0.87 & 0.04 \\
$2.1-4$ & -0.33 & 0.08 & 0.71 & $<0.01$ \\
$4.1-6$ & -0.55 & 0.08 & 0.57 & $<0.01$ \\
$6.1-8$ & & & & \\
Agonistas (total) & & & & \\
Horas de viaje & Ref. & Ref. & Ref. & \\
$0-2$ & 0.65 & 0.29 & 1.9 & 0.03 \\
$2.1-4$ & 1.28 & 0.29 & 3.6 & $<0.01$ \\
$4.1-6$ & 1.20 & 0.25 & 3.3 & $<0.01$ \\
$6.1-8$ & & & & \\
\hline
\end{tabular}

${ }^{1} \mathrm{RR}$ (riesgo relativo)

${ }^{2}$ Ref (categoría considerada de referencia en el análisis)

De acuerdo con la estimación de los parámetros de los modelos de la distribución de Poisson (Cuadro 3), se estableció que, los novillos tuvieron 4.5 veces más riesgo de caer durante las dos últimas horas de viaje en comparación con las dos primeras horas $(\mathrm{RR}>1$, $\mathrm{p}<0.05)$. Los comportamientos afiliativos fueron constantes durante el viaje $(\mathrm{RR}<1$; $\mathrm{p}<0.05$ ). Es posible que el aumento de la probabilidad de las caídas durante las dos últimas horas del viaje esté asociado con las características topográficas de la vía, teniendo en cuenta que este trayecto correspondió al de montaña, caracterizado por curvas pro- nunciadas y un aumento rápido de la altitud sobre el nivel del mar. Otros factores involucrados pudieron ser el cansancio de los animales, los cambios inesperados en la velocidad del vehículo, la falta de entrenamiento y conocimiento del conductor de cómo transportar adecuadamente el ganado (Tarrant y Grandin, 2000). Estos factores, asimismo, han sido descritos como causa de accidentalidad durante el transporte de animales (Miranda-de la Lama et al., 2011) y por su efecto negativo en la estabilidad y posibilidad de descanso de ovejas durante el viaje (Cockram et al., 2004; Fox et al., 2014). 
Cuadro 4. Descripción de indicadores fisiológicos de estrés durante el transporte de ocho horas de novillos, de acuerdo con su ubicación en el camión

\begin{tabular}{lcc}
\hline $\begin{array}{l}\text { Variables sanguíneas } \\
\text { (Media } \pm \text { d.e. })\end{array}$ & Compartimento anterior & Compartimento posterior \\
\hline Cortisol $(\mu \mathrm{g} / \mathrm{dl})$ & $175.3 \pm 70.6^{\mathrm{a}}$ & $183.6 \pm 48.0^{\mathrm{b}}$ \\
VGA $(\%)$ & $53.6 \pm 1.5$ & $54.3 \pm 1.8$ \\
Glucosa $(\mathrm{g} / \mathrm{l})$ & $8.07 \pm 1.6$ & $8.1 \pm 1.7$ \\
Proteína total $(\mathrm{g} / \mathrm{l})$ & $86.1 \pm 7.3$ & $85.7 \pm 4.9$ \\
Albúmina $(\mathrm{g} / \mathrm{l})$ & $38.1 \pm 0.5$ & $40.4 \pm 0.9$ \\
Creatinina $(\mathrm{mmol} / \mathrm{l})$ & $167.4 \pm 17.0$ & $166.2 \pm 16.9$ \\
Urea $(\mathrm{mmol} / \mathrm{l})$ & $9.1 \pm 2.6^{\mathrm{a}}$ & $10.9 \pm 4.1^{\mathrm{b}}$ \\
Creatinquinasa $(\mathrm{U} / \mathrm{l})$ & $993.0 \pm 362.5$ & $1.033 \pm 454$ \\
$\beta$ HB $(\mathrm{mmol} / \mathrm{l})$ & $0.41 \pm 0.43^{\mathrm{a}}$ & $0.30 \pm 0.15^{\mathrm{b}}$ \\
Lactato $(\mathrm{mg} / \mathrm{dl})$ & $4.37 \pm 1.4^{\mathrm{a}}$ & $6.0 \pm 3.6^{\mathrm{b}}$ \\
Leucocitos $(\mathrm{mil} /)$ & $243.8 \pm 10.9$ & $234.5 \pm 9.6$ \\
Neutrófilos/Linfocitos $(\mathrm{N} / \mathrm{L})$ & $0.79 \pm 0.06$ & $0.8 \pm 0.07$ \\
\hline
\end{tabular}

Las variables fueron transformadas previas al análisis estadístico

${ }^{a, b}$ Superíndices diferentes dentro de filas indican diferencias significativas $(p<0.05)$

Los cambios fisiológicos observados (Cuadro 4) que no comprometieron el metabolismo proteico, ni los constituyentes celulares sanguíneos, indican que los novillos mantuvieron la homeostasis por factores relacionados con su buena condición física y adaptabilidad (Cockram et al., 2004), el diseño del vehículo (Lambooij et al., 2012), las condiciones del viaje, la ausencia de mezcla social (Mounier et al., 2005) y, posiblemente por otros factores que requieren ser evaluados en estudios posteriores. De igual manera, los niveles sanguíneos de $\beta \mathrm{HB}$ encontrados, acordes con los parámetros basales para la especie (Kaneko et al., 2008), sugieren que los bovinos requieren varios días de ausencia de alimento para experimentar ayuno fisiológico (Tadich et al., 2003).

El incremento de las concentraciones sanguíneas de cortisol, glucosa y VGA, independiente de su localización en el camión, puede ser una respuesta a la adaptación de los animales a la novedad y a la demanda física adicional del transporte y el manejo presacrificio, resultados similares a los reportados en Colombia con novillos cebú bajo similares condiciones, pero sometidos a tiempos de transporte inferiores a $4 \mathrm{~h}$. Estos resultados sugieren que tanto los viajes de corta y larga duración se convierten en un desafío fisiológico para los bovinos (Early et al., 2012; Romero et al., 2014).

En concordancia con lo expuesto por van de Water et al. (2003), se observaron diferencias significativas en la respuesta fisiológica al estrés de los bovinos de acuerdo con la localización en el camión (Cuadro 4). Los novillos ubicados en el compartimento posterior presentaron niveles séricos más altos de cortisol y lactato, debido posiblemente a la necesidad de realizar un mayor esfuerzo físico para mantener el balance (Hambrecht et al., 2005; Boles et al., 2015). Además, este compartimento al estar más retirado del cen- 
tro de gravedad del vehículo es más inestable y se ve más afectado por las corrientes de aire. Los altos niveles de CK indican daño de las células musculares (Romero et al., 2014), en concordancia con la alta frecuencia de pérdidas de balance en el estudio.

El $\mathrm{pH}$ medido a las $24 \mathrm{~h}$ del sacrificio $\left(\mathrm{pH}_{24}\right)$ es el indicador más importante usado en la industria para evaluar la calidad de la carne a nivel comercial, por su efecto en el color, textura, terneza y capacidad de retención de agua (Dunne et al., 2011). En este estudio, el $63.5 \%(n=36)$ de las canales presentó $\mathrm{pH}_{24} \geq 5$.8, sin observarse diferencias significativas de acuerdo con la ubicación de los novillos en el camión. Es posible que el esfuerzo físico de los novillos para mantener el balance durante el viaje y para responder a la novedad del presacrificio, haya provocado un aumento del metabolismo oxidativo y el agotamiento de las reservas de glucógeno muscular (Archile-Contreras y Purslow, 2011; Boles et al., 2015). Sin embargo, este proceso metabólico es multicausal y está influenciado por factores intrínsecos (raza, edad, sexo, peso y temperamento de los animales) y extrínsecos (sistema de producción, dieta, interacción humano-animal, método de aturdimiento, época del año, tipo de oreo, refrigeración de la carne, entre otros) (Ferguson y Warner, 2008).

\section{Conclusiones}

- El transporte comercial con una duración de $8 \mathrm{~h}$ fue un factor de estrés fisiológico para los novillos, con implicaciones negativas en la presencia de carne con $\mathrm{pH}$ alto.

- La ausencia de mezcla social favoreció el comportamiento social positivo, que promovió la cohesión y afiliación entre congéneres.

- Los novillos localizados en el compartimento posterior del camión presentaron niveles más altos de cortisol y lactato como respuesta al mayor esfuerzo físico para mantener el balance.

\section{Agradecimientos}

Los autores agradecen a la Vicerrectoría de Investigaciones y Postgrados de la Universidad de Caldas por la financiación del trabajo y al personal del frigorífico Frigocentro por el apoyo logístico.

\section{Literatura Citada}

1. Archile-Contreras AC, Purslow PP. 2011. Oxidative stress may affect meat quality by interfering with collagen turnover by muscle fibroblast. Food Res Int 44: 582-588. doi: 10.1016/j.foodres. 2010.12.002

2. Boissy A, Manteuffel $G$, Jensen MB, Moe RO, Spruijt B, Keeling LJ, Winckler C, et al. 2007. Assesment of positive emotions in animals to improve their welfare. Physiol Behav 92: 375397. doi: 10.1016/j.physbeh.2007.02.003

3. Boles JA, Kohlbeck KS, Meyers MC, Perz KA, Davis KC, Thomson JM. 2015. The use of blood lactate concentration as an indicator of tempe-rament and its impact on growth rate and tenderness of steaks from Simmental X Angus steers. Meat Sci 103: 68-74. doi: 10.1016/j.meatsci. 2015.01.003

4. Cafazzo S, Magnani D, Calà P, Razzuoli E, Gerardi G, Bernardini D, Amadori M, Nanni Costa L. 2012. Effect of short road journeys on behaviour and some blood variables related to welfare in young bulls. Appl Anim Behav Sci 139: 26-34. doi: 10.1016/ j.applanim.2012.03.009

5. Cockram MS, Baxter EM, Smith LA, Bell S, Howard CM, Prescott RJ, Mitchell MA. 2004. Effect of driver behaviour, driving events and road type on the stability and resting behaviour of sheep in transit. Anim Sci 79: 165-176. doi: 10.1017/S1357729800054631

6. Dunne PG, Monahan FJ, Moloney AP. 2011. Current perspectives on the darker beef often reported from extensively-managed cattle: does physical activity 
play a significant role? Livestock Sci 142: 1-22. doi: 10.1016/j.livsci.2011.06.018

7. Early E, Murray M, Prendiville DJ, Pintado B, Borque C, Canali E. 2012. The effect of transport by road and sea on physiological, immunity and behavior of beef cattle. Res Vet Sci 92: 531-541. doi: 10.1016/j.rvsc.2011.04.002

8. Ferguson DM, Warner RD. 2008. Have we underestimated the impact of pre-slaughter stress on meat quality in ruminants? Meat Sci 80: 12-19. doi: 10.1016/j.meatsci.2008.05.004

9. Fox J, Widowski T, Torrey S, Nannoni E, Bergeron R, Gonyou $\mathrm{HW}$, Brown $J A$, et al. 2014. Water sprinkling market pigs in a stationary trailer. 1 . Effects on pig behaviour, gastrointestinal tract temperature and trailer micro-climate. Livestock Sci 160: 113-123. doi: 10.1016/ j.livsci.2013.12.019

10. Gallo C, Lizondo G, Knowles TG. 2003. Effects of journey and lairage time on steers transported to slaughter in Chile. Vet Rec 152: 361-364. doi: 10.1136/ vr.152.12.361

11. Hambrecht E, Eissen JJ, Newman DJ, Smits CH, den Hartog LA, Verstegen MWA. 2005. Negative effects of stress immediately before slaughter on pork quality are aggravated by suboptimal transport and lairage conditions. J Anim Sci 83: 440-448. doi: $10.2527 / 2005.832440 \mathrm{x}$

12. Kaneko J, Harvey J, Bruss M. 2008. Clinical biochemistry of domestic animals. $6^{\text {th }}$ ed. San Diego, USA: Academic Press. 484 p.

13. Kannan G, Kouakou B, Terril TH, Gelaye S. 2003. Endocrine, blood metabolite, and meat quality changes in goats as influenced by short-term, preslaughter stress. J Anim Sci 81: 14991507. doi: $10.2527 / 2003.8161499 x$

14. Lambooij E, van Der Werf, JTN, Reimert HGM, Hindle VA. 2012. Compartment height in cattle transport vehicles. Livestock Sci 148: 87-94. doi: 10.1016/j.livsci.2012.05.014
15. Magnani D, Cafazzo S, Calà P, Razzuoli E, Amadori M, Bernardini D, Gerardi G, Nanni Costa L. 2014. Effect of long transport and environmental conditions on behavior and blood parameters of postweaned piglets with different reactivity to backtest. Livestock Sci 162: 201-208. doi: 10.1016/ j.livsci.2014.01.011

16. Mather AE, Innocent GT, McEwen $S A$, Reilly WJ, Taylor DJ, Steele WB, Gunn GJ, et al. 2007. Risk factors for hide contamination of Scottish cattle at slaughter with Escherichia coli O157. Prev Vet Med 80: 257-270. doi: 10.1016/ j.prevetmed.2007.02.011

17. Millman ST, Duncan IJH. 2001. Social cognition of farm animals. In: Keeling L, Gonyou H (eds). Social behaviour of adaptation and domestication in livestock. Wallingford, UK: CABI Publishing. p 373-400.

18. Miranda-de La Lama GC, Mattiello GC. 2010. The importance of social behavior for goat welfare in livestock farming. Small Ruminant Res 90: 1-10. doi: 10.1016/j.smallrumres.2010.01.006

19. Miranda-de La Lama GC, Sepúlveda WS, Villarroel M, Olleta, JL, GarcíaBelenguer S, Maria GA. 2011. Livestock vehicle accidents in Spain: causes, consequences, and effects on animal welfare. J Appl Anim Welf Sci 14: 109-123. doi: 10.1080/10888705.2011.551622

20. Miranda-de La Lama GC, Villarroel M, María GA. 2012. Behavioural and physiological profiles following exposure to novel environmental and social mixing in lambs. Small Ruminant Res 103: 158163. doi: 10.1016/j.smallrumres.2011.08.007

21. Mounier L, Veissier I, Boissy A. 2005. Behavior, physiology, and performance of bulls mixed at the onset of finishing to form uniform body weight groups. J Anim Sci 83: 1696-1704. doi: 10.2527/ 2005.8371696x 
22. Romero MH, Gutiérrez C, Sánchez JA. 2012. Evaluation of bruises as an animal welfare indicator during preslaughter of beef cattle. Rev Colomb Cienc Pecua 25: 267-275.

23. Romero MH, Uribe-Veláquez LF, Sánchez JA. 2014. Physiological profiles of Zebu steers during transport and pre-slaughter. Rev Colom Cienc Pecua 27: 282-289.

24. Romero MH, Uribe-Velásquez LF, Sánchez JA, Miranda-de La Lama GC. 2013. Risk factors influencing bruising and high muscle $\mathrm{pH}$ in Colombian cattle carcasses due to transport and pre-slaughter operations. Meat Sci 95: 256-263. doi: 10.1016/ j.meatsci.2013.05.014

25. Romero MH, Uribe-Velásquez LF, Sánchez JA, Rayas-Amor AA, Miranda-de La Lama GC. 2017. Conventional versus modern abattoirs in Colombia: impacts on welfare indicators and risk factors for high muscle $\mathrm{pH}$ in commercial Zebu young bulls. Meat Sci 123: 173-181. doi: 10.1016/j.meatsci. 2016.10.003

26. Stockman CA, Collins T, Barnes AL, Miller D, Wickham SL, Beatty DT Blache D, et al. 2013. Flooring and driving conditions during road transport influence the behavioural expression of cattle. Appl Anim Behav Sci 143: 18-30. doi: 10.1016/j.applanim.2012.11.003

27. Tadich N, Gallo C, Echeverría R, van Schaik G. 2003. Efecto del ayuno durante dos tiempos de confinamiento y de transporte terrestre sobre algunas variables sanguíneas indicadoras de estrés en novillos. Arch Med Vet 2: 171-185. doi: 10.4067/S0301-732X2003000200005

28. Tarrant V, Grandin T. 2000. Cattle transport. In: Grandin T. (ed). Livestock handling and transport. $2^{\text {nd }}$ ed. Wallingford, UK: CABI Publication. $p$ 151-173.

29. Temple D, Manteca X, Velarde A, Dalmau A. 2011. Assessment of animal welfare though behavioural parameters in Iberian pigs in intensive and extensive conditions. Appl Anim Behav Sci 131: 29-39. doi: 10.1016/ j.applanim.2011.01.013

30. van De Water G, Verjans F, Geers $R$. 2003. The effect of short distance transport under commercial conditions on the physiology of slaughter calves; $\mathrm{pH}$ and colour profiles of veal. Livestock Prod Sci 82: 171-179. doi: 10.1016/ S0301-6226(03)00010-1 